réglage adopté pour le relais, le déclenchement a pu se produire pour une résistance atteignant 50000 ohms environ.

Dans chacune des trois expériences effectuées, le courant passant dans le relais était largement suffisant pour obtenir la fermeture du circuit auxiliaire alimenté par la batterie d'acculateurs sur la bobine du disjoncteur, et l'ouverture de cet appareil se produisait au bout d'un temps absolument inappréciable.
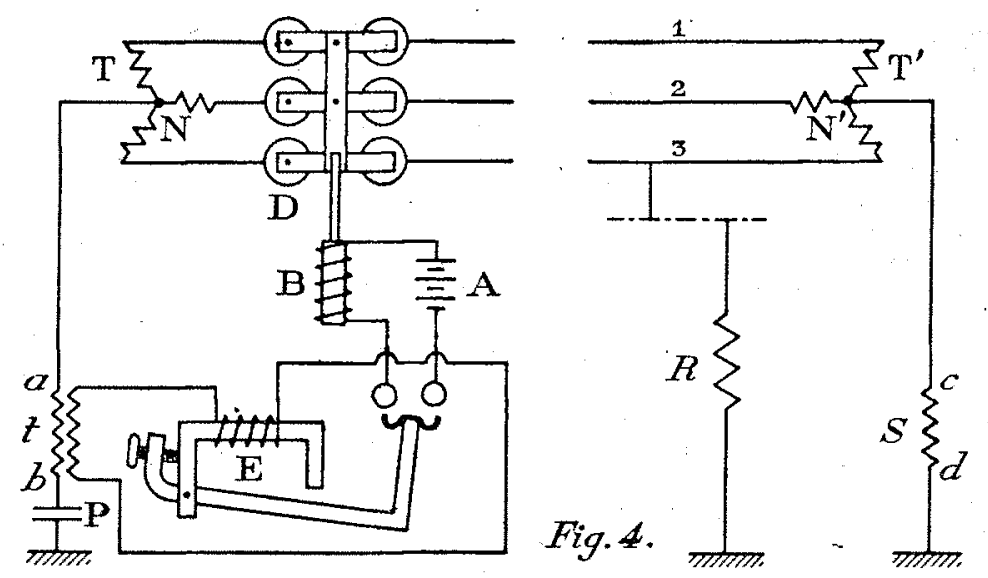

Si l'un des conducteurs était venu en contact avec un fil téléphonique ou télégraphique relié au sol par l'intermédiaire d'un parafoudre à faible distance d'éclatement, ce parafoudre aurait infailliblement cédé sous l'influence de la différence de tension élevée, produite entre ce fil téléphonique ou télégraphique et le sol.

Il en serait résulté, pour le fil correspondant de la camalisation à haute tension, une mise à la terre presque franche, amenant infailliblement le déclanchement du disjoncteur.

Dans d'autres essais, effectués en octobre, la ligne considérée, alimentée par. le courant monophasé à 2800 volts, et 53 périodes du secteur de l'Onest-Lumière, était munie à son origine d'un point neutre constitué, pour la facilité des essais, par les primaires de deux transformateurs placés en série et à circuits secondaires ouverts; dans ces appareils, d'une puissance de $1 \mathrm{kw}$ environ, la self-induction du circuit primaire avait été diminuée en créant, à l'aide de cales de bois, des entrefers dans le circuit magnétique. Un point neutre avait été constitué, d'une manière analogue, à l'extrémité de la ligne. Le transformateur branché entre le point neutre d'origine et la terre avait une puissance de $200 \mathrm{w}$ environ, avec un rapport de transformation égal à 5. Le disjoncteur était un appareil bipolaire à rupture dans l'huile, dont la bobine était alimentée par du courant alternatif à 110 volts, le circuit alimentant cette bobine pouvait être fermé par le relais à sensibilité réglable. Des coupe-circuits placés sur chacun des fils de ligne permettent de simuler une rupture de ces fils, et une dérivation prise par l'un d'entre eux permettait de simuler un contact accidentel avec la terre.

Daus chacun de ces cas, le fonctionnement du disjoncteur avait lieu sans aucune difficulté réalisant une interruption instantanée du courant sur la ligne.

Il résulte, de mesures effectuées ultérieurement, que le relais, réglé pour sa sensibilité maxima, pouvait fonctionner à partir d'une tension aux bornes de 10 volts, correspondant à une tension de 50 volts aux bornes du primaire du transformateur de terre. Pour obtenir cette tension minima, en cas de rupture de l'un des fils, on doit employer des points neutres dont la self-induction correspond $\ddot{a}$ une consommation de 125 volts-amperes sous
2800 volts En pratique, ces points neutres auraient été constitués par des bobines de self-induction à circuit magnétique ouvert, pour lesquelles la valeur de $\cos$ o aurait été plus réduite, ce qui aurait permis de diminuer encore la puissance absorbéc, tout en conservant un courant sulfisant pour lalimentation du relais.

M. P.

\section{Historique de la Question des Patentes des Usines électriques}

Tous les industriels connaissent aujourd'hui la loi de Finances pour l'année 1906 (promulguée le I9 juillet 1905, publiée à l'Officiel du 20, applicable à partir du r ${ }^{\text {or }}$ janvier Igo6), qui a modifié profondément la question des patentes des usines électriques. Ils ont certainement lu avec plaisir, non seulement le texte de la loi, mais encore la circulaire de la Direction générale des Contributions directes qui explique nettement :

I. - Que les usines de production ne seront taxées au droit fixe que sur l'eurs machines de production et non pas sur leurs transformateurs.

II. - Que l'éclairage d'une usine, obteinu par la transformation en lumière d'une partie de l'énergie fournie à l'usine par un autre établissement industriel, ne donnera pas lieu à l'application du tarif-lumière, mais se confondra pour la perception, avec le droit dû en général pour la puissance des moteurs installés.

Quelques lecteurs nous ont demandé de faire l'exposé complet de ce qui s'est passé, de la situation qui a menacé pendant un moment l'industrie électrique, et de celle qui; aujourd'hui, se présente à elle. Tel est le but de la présente note.

\section{I}

Lorsqu'une industrie nouvelle voit le jour, son extrait de naissance officiel lui est donné par le Gouvernement.... sous la forme d'une feuille d'impôt; toutefois, cet enregistrement authentique, qui n'a rien de bien réjouissant, ne va pas sans difficulté. Lorsqu'une profession est indiquée aux tableaux annexés à la loi sur les patentes, il n'y a qu'une simple référence à donner, et la feuille se trouve rapidement établie. Le contrôleur indique les mots sacramentels : "Loi du.... article $n^{\circ}$...... Tableau.... ". Mais il n'en est pas de même quand, postérieurement au dernier texte législatif, la nouvelle profession arrive au monde. Le principe de la généralité des patentes exige qu'on assujettisse non seulement ceux qui exercent une des professions prévues dans le tarif, mais encore tous. ceux qui se livrent à des commerces ou industries non dénommés, en les classant par analogie dans la même catégorie que les industries similaires. (Loi de Brumaire, an VI). Le pouvoir compétent pour décider de l'analogie est celui du Préfet qui prend un "arrêté d"assimilation " (loi de I880).

Mais il serait contraire au principe de notre droit de donner au Préfet un pouvoir permanent de classement; ce qui constituerait un empiètement grave sur l'autorite législative du Parlement : aussi la loi n'a-t-elle pas tardé à ajouter ce correctif : "Tous les cinq ans, des tableaux additionnels conte" nant la nomenclature des commerces, industries et profes" sions classées par voie d'assimilation depuis trois années " au moins, seront soumis à la sanction législative ".

Ainsi donc, l'arrêté d'assimilation du Préfet n'est que provisoire; et même, dans le délai de cinq ans pendant lesquels il attend la saction législative, il n'est qu'exécutoire, mais il n'est pas inattaquable; c'est-à-dire que le contribuable peut se pourvoir devant la juridiction compétente (Conseil de Préfecture et Conseil d'Etat), pour faire déclarer fausse l'assimilation qui le frappe. S'il triomphe, le Préfet doit prendre un nouvel arrêté de classement sur d'autres bases; mais tout cela en pra- 
tique est très rare. Tout le monde est d'accord pour reconnâ̂tre que les arrêtés d'assimilation sont généralement adoptés par le tribunal administratif. Il faut vraiment qu'il y ait des erreurs colossales pour ouvrir les yeux des juges qui, en grande majorité, se rapportent aux études préalables de l'Administration compétente : comme type des arrêtés d'assimilation cassés par le Conseil d'Etat, on cite quelquefois celui qui avait été pris par un Préfet pour assimiler à un distributeur de gaz d'éclairage le propriétaire qui avait vendu une source d'eau vive à une commune, à la condition qu'il n'en ferait pas la distribution!! On peut citer aussi le cas du curé qui était imposé comme marchand de cire, pour avoir une fois donné des cierges à ses paroissiens! Il est évident qu'un tribunal ne pouvait laisser passer de pareilles énormités.... mais, dans maintes occasions, il a omis de rectifer certaines erreurs dont la correction aurait pu cependant être intéressante.

Si l'arrêté est généralement bien vu par l'Administration, cela provient de ce que, en général, il tranche dans le sens de la taxation la plus élevée toutes les difficultés résultant de la fixation exacte de l'analogie entre la profession nouvelle et telle autre catégorie existante. Mais le courant de sympathie est encore bien plus remarquable entre l'arrêté d'assimilation et le pouvoir législatif qui, tous les cinq ans, le révise théoriquement, et pratiquement, dans la plupart des cas, le classe purement et simplement dans un texte définitif et en fait, en un mot, une loi formelle. Cette manœuvre se fait en quelque sorte automatiquement. L'examen auquel les Chambres procèdent est toujours très aride, et pour discuter sur ces questions techniques avec le Ministre des Finances et surtout avec le Directeur général des Contributions qui connaît d'une façon merveilleuse les rouages compliqués de son Administration et remplit toujours les fonctions de Commissaire du Gouvernement, il faudrait des orateurs habitués à ce genre d'études. Si bien que c'est toujours une chose grave de voir un arrêté préfectoral frapper une industrie nouvelle : neuf fois sur dix, la taxation provisoire sera demain la taxation définitive. On remarquera, d'ailleurs, qu'il y a entre la décision du Préfet et la sanction des Chambres une différence caractéristique. Le Préfet doit toujours, quand il prend son arrêté, dire qu'il agit en vertu d'une analogie qu'il vise spécialement dans son texte, sous peine de faire une œuvre illégale. Le pouvoir législatif n'a pas même à connaître ce scrupule. On sait, en effet, comment les choses se passent : tous les cinq ans (I) la loi des patentes doit être revisée; le projet de loi qui contient les professions nouvelles, classées ainsi que nous venons de le dire, dans la catégorie où les Préfets les ont placées, est dis. tribué aux Députés. S'ils l'adoptent, la loi est souveraine, alors même que l'analogie serait fausse; car s'il y a une chose que l'on ne puisse reprocher à la loì, c'est évidemment d'être illégale.

Cette genèse de l'assimilation, très simple pour le petit nombre des industriels qui la connaissent sans en parler, doit être condensée en une phrase à l'usage de ceux, beaucoup plus nombreux, qui en parlent sans la connaitre. L'assimilation en résumé, naît forcément avec la profession; juridiquement elle est temporaire; habituellement, sauf de rares exceptions, elle devient défnitive; théoriquement, elle peut être attaquée deux fois : une première fois par la partie frappée de la taxation devant le Tribunal; une seconde fois, devant la Chambre, au moment de la révision, par le groupement des intéressés au Parlement; pratiquement, elle persiste dans la majorité des cas.

\section{II}

Que s'est-il passé pour les professions de Producteurs d'Electricité? Le courant peut être utilisé sous deux formes:

(1) Les deux premières ruvisions se sont faites à l'heure : la dernière a trấné dix ans à cause des difficultés soulevées au sujet de la taxe des coopératives et des grands magasins. la lumière et la force motrice. Lorsque la loi des patentes de I 890 parut, le tableau qui y était annexé ne prévoyait que la lumière (I); si bien que, vers 1900, l'Administration se crut autorisée à prendre un arrêté d'assimilation pour les usines produisant l'énergie électrique. Une catégorie pouvait la tenter : celle de loueur de Force motrice : on sait, en effet, qu'au tableau A, $6^{\circ}$ classe, figure cette profession ainsi visée : Force motrice (loueur de); celui qui, possesseur de bâtiments et de moteurs, qu'dl n'emploie pas pour son propre compte, les loue $\grave{a}$ divers industriels auxquels $i l$ s'engage à fournir une force molrice determinie.

$$
\text { Droit proportionnel }\left\{\begin{array}{c}
\text { sur la maison d'habitation . . } 30^{\mathrm{me}} \\
\text { sur les locaux servant a l'exer- } \\
\text { cice de la profession. . . . } 40^{\mathrm{me}}
\end{array}\right.
$$

Mais cette catégorie, comme on le voit, nimpose que les locaux servant à l'exercice de la profession; c'est-à-dire, en réalité, le réduit où est logé la machine à vapeur, tandis que la tarification du tableau $C$ visant les usines d'éclairage, impose l'établissement industriel, c'est-à-dire la valeur complète de l'immeuble et de tout l'outillage (du contenant et du contenu) et en plus demande un droit fixe de I franc.

Aussi tous les Préfets intéressés (Rhône, Isère, les Alpes, Savoie) prirent les mêmés arrêtés d'assimilation, comme si le mot magique "électricité " était seul à entrer en ligne de compte, l'utilisation qui en était faite ne paraissant qu'au second plan, ou même pas du tout. La lumière électrique, objet utile, mais de luxe, et la force, objet indispensable et se payant moins cher, furent confondues dans une même taxe. Des protestations se manifestèrent de suite; l'une d'elles fut portée par la Compagnie des Forces motrices du Rhône, devant le Conseil de Préfecture, suivant la procédure que nous indiquons plus haut; elle fut rejetée le 30 décembre I9O2 par arrêté formel et l'assimilation de la production de l'électricité énergie à l'électricité lumière fut confirmée.

La Compagnie se pourvut devant le Conseil d'Etat et le monde des électriciens attendait, sans grande espérance, l'issue du pourvoi, lorsqu'un fait de bien plus grande importance excita ses appréhensions.

\section{III}

A peu près au même moment, on annonça la révision quinquennale par la Chambre des Députés du tarif des patentes et le projet de la loi annoncée portait à l'article "électricité " un texte qui devait faire ouvrir les yeux même aux moins éclairés. Au lieu de reproduire les termes de la loi de I890, savoir : Electricité (Exploitant une usine d'éclairage électrique par l'), on pouvait lire : Electricité (exploitant une usine pour la production de l'), le même droit fixe de 1 franc, et le même droit proportionnel étant exigés pour les deux.

La sanction législative allait donc intervenir, et la loi allait couper court, par un argument sans réplique, à toute discussion, consacrant une assimilation qui est la négation de toute idée pratique; pour faire adopter l'énergie électrique et faire reléguer à la vieille ferraille la machine à vapeur dont l'industrie s'est toujours servie, il faut que le producteur d'électricité offre un pris aussi réduit que possible, le distributeur de lumière, dont il ne faudrait pas toutefois exagérer les bénéfices, est cependant dans une situation un peu préférable. Sans doute, la jurisprudence du Conseil d'Etat quí admet le

(I) Le tableau hien connu des électriciens porte :

Tabreat C. - Electricité (Exploitant une usine d'éclairage par l') i fr. le kilowatt ou fraction de kilowat de la puissance untle des machines dynamo-électriques fonctionnant simultanément.

Les conduites et. les câbles extérieurs n'entreront pas dans l'estimation de la valeur locative.

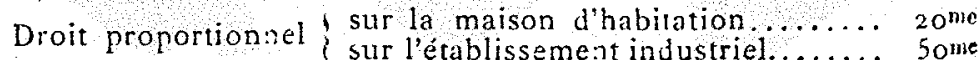


remplacement du concessionnaire gazier dans le seul cas où la lumière électrique revient à meilleur marché que le bec Auer, lui fait une situation souvent terrible; mais néanmoins, il peut encore faire admettre des prix qui lui assurent plus que ses frais généraux.

Lorsque le projet de loi vint à la Chambre, deux orateurs s'inscrivirent pour prendre la parole. M. Cazeneuve, Député de Lyon, et M. de Ramel demandèent : le premier que le principe d'une différence dans la taxation fut indiquée dans la. lo $;$; le second, que la base même de la taxe fut modifiée: Tous les deux, avec leur grande habitude de la vie parlemrentaire, comprirent qu'il serait souverainement imprudent de demander que la taxe du loueur de force motrice fut étendue à la distribution de l'énergie électrique et que ce serait courir à un échec certain. M. de Ramel a expliqué d'une façon très serrée que, pour luí, les arrêtés d'assimilation, pris pour faire rentrer l'énergie électrique dans la même catégorie que l'éclairage, n'étaient pas valables, puisqu'il existait au tarif une catégorie bien plus voisine, celle de loueur de force motrice; et il ajoutait que si, pour l'avenir, l'Administration se refuscit $\grave{a}$ assinniler l'énergie électrique à la force motrice, elle devait faire une taxation nouvelle, intermédiaire, au lieu d'adopter d'un seul coup le tarif que l'éclairage lui-même a déjà de la peine à supporter.

"Dans l'état de notre législation, disait le Député du Gard, "avec l'assentiment plusieurs fois répété du Député du "Rhône, la production de l'énergie électrique est assimilable " à l'industrie de location de force motrice, ou plus exacte" ment, rentre dans cette catégorie.... Sans doute, les progrès " de la science permettent de transporter ajourd hui au loin " cette énergie, notamment par l'électricité et l'air comprimé. "Si vous avez voulu frapper cette nouvelle force portée à "distance qui, par sa transformation, sert de force motrice à " diverses industries, je serais disposé à l'admettre, mais il " s'agit de savoir, en ce cas, si vous la frappez pour ce qu'elle " est ou pour ce qu'elle n'est pas.... ".

\section{IV}

A cela le Ministre des Finances répondit, - prenant la parole lui-même, bien que le Commissaire du Gouvernement eût jusqu'à ce moment soutenu le poids de la discussion, qu'il reconnaissait toute l'importance de la question soulevée; qu'il était désireux de ménager une industrie particulièrement intéressante entre toutes, mais qu'il fallait, pour cela, faire une étude noukelle. On convint danc de ne pas légiférer sur la question de la force motrice et de laisser subsister pour le moment l'ancien tarif exclusivement relat if à l'éclairage, sauf au Ministre à étudier la question de la force motrice.

Les paroles de M. Rouvier sont les suivantes : « M. de Ramel " se plaint, comme s'était plaint M. Cazeneuve, que le projet " ne distingue pas entre l'électricité destinée à l'éclairage et "I'électricité destinée à la force motrice. Je reconnais très vo"lontiers l'importance de la question soulevée: Le Gouverne" ment a le devorr de n'entraver en rien, de ne pas retarder " par des lois fuscales exagérées, le développement d'une in"dustrie nouvelle qui apparâ̂t comme pouvant peut-être " transformer le régime industriel de notre pays. Je vais faire "êtudier la question et je vous proposerai un projet spécial" $\%$

Ces simples paroles, dont on comprend l'importance capitale, quand on sait avec quelle mesure et quelle modération parlent les Ministres des Finances au sujet de l'atténuation possible d'une taxe, ont eu un double effet : en premier lieu, elles permettaient d'espérer une taxation nouvelle, adoucie par rapport à l'ancienne; comme le demandaient avec une ardente conviction M. Cazeneuve et après lui M. Ramel; en second lieu, elles laissaient comprendre qu'en réalité, l'Administration des Finances, représentée par son chef hiérarchique, était d'accord avec eux, en principe, sur l'illégalité de l'assimilation.

\section{V}

Aussi, deux résultats ont-ils été obtenus.

Le plus important, celui qui concernait l'avenir, s'est révélé dans la loi de Finances pour 1906, figurant à l'Officiel du 20 juillet 1905 .

La gestation de cette loi a été laborieuse et l'étude a été longue. Sans parler des personnes dévouées résidant à Paris, par conséquent à proximité du Ministère, qui y ont porté à maintes reprises les réponses aux questions qui leur étaient posées, sans parler de celles non moins dévoueés qui, habitant dans le pays de la Houille Blanche, se sont déplacées bien des fois pour la caurse commune, se rendant à la convocation de lal Commission des Patentes, quel est l'industriel, l'ingénieur un peu en vue, le concessionnaire d'une ville importante qui n'a pas reçu la visite des contrôleurs et inspecteurs spéciaux nommés par le Ministère pour se faire une opinion.

Tout cela a abouti au texte que l'on connaît : Electricité : Exploitant une usine pour la praduction ou La transformation de $l$ :

go centimes par kitlowatt ou fraction de kilowatt de la puissance utile des machines ou appareils de production ou de transformation, non compris les machines ou appareils de secours.

Ce droit serct réduit :

I $^{\circ}$ A 60 centimes pour les établissements à l'égard desquels il sera justifié que la puissance des appareils d'éclairage n'excède pas les sept diaièmes de la puissance totale des moteurs et appareils de toute nature installés chez les clients:

$2^{\circ} A$ jo centimes pour les établissements à l'égard desquels il sera justifié que la puissance des appareils d'éclairage n'excède pas le dizième de la puissance totale des moteurs et appareils de toute nature installés chez les clients.

Les conduites et câbles extéxieurs n'entreront pas dans l'estimation de la valeur locative.

Nous ferons remarquer : $I^{\circ}$ que le texte exclut la possibilité d'imposer, chez le producteur de l'électricité, les transformateurs, en plus des organes générateurs de l'électricité, puisque le mot "ou. " est placé justement pour empêcher une addition de ces deux sortes d'appareils; $2^{\circ}$ Que, non seulement, lénergie électrique n'est plus imposée comme l'éclairage, mais que celui-ci jouit d'une petite diminution ( 90 centimes an lieu de I franc).

En réalité, ce résultat ramène à une situation plus voisine de l'équité les distributeurs d'énergie qui payaient I franc en vertu des anciens arrêtés d'assimilation, et surtout il a eu pour effet d'écarter le danger d'un texte législatif consacrant pour toujours cette assimilation.

C'est beaucoup, en comparaison de ce que l'on pouvait craindre. Il est évident que c'est peu, si l'on se place au point de vue de quelques industriels qui avaient interprété la bonne volonté promise par le Minsitre, en ce sens que l'énergie élecirique ne paierait plus rien; mais la conséquence extrême de cet état d'esprit nous amènerait à demander au Ministre la suppression des impôts : système auquel nous ne verrions aucun inconvénient s'il n'était pas une utopie:

\section{VI}

Pour apprécier le second résultat, il faut descendre de la sphère élevée du domaine législatif et se cantonner dans celle: plus prosaïque du domaine judiciaire.

Nous avons dit plus haut que les arrêtés de Préfecture validant les arrêtés d'assimilation, avaient été frappés d'un pourvoi au Conseil d'Etat. Il est peu probable que cette haute juridiction se fût départie de ce qu'elle a fait si souvent, et n'eût validé l'arrêté, si la séance du 23 décembre 1903 n'était pas intervenue auparavant; mais, du moment que le Ministre lui-même n'avait accepté cet arrêté d'assimilation que lui si- 
gnalaient MM. Cazeneuve et de Ramel qu'avec une réserve calculée, du moment qu'il ne voulait pas en faire état pour l'avenir, il est évident. que le Conseil d'Ltat devait prendre en considération les arguments excellents que les demandeurs au pourvoi lui faisaient valoir. On peut les résumer ainsi : l'Administration préfectorale a taxé comme électricité-lumière, l'électricité-force; elle devait seulement signaler au pouvoir central qu'il n'existait pas de catégorie répondant d'une façon directe à l'industrie nouvelle; mais en l'absence de tottte taxation indiquée par la loi, elle devait rechercher la catégorie la plus voisine. Or, celle-ci ne peut être que la catégorie des loueurs de force motrice, en attendant la loi nouvelle. Quant à l'industrie produisant la lumière et la force, il y aura un examen préaliable à faire, d'accord avec elle et, sous la réserve d'en saisir le Conseil de Préfecture, l'Administration recherchera : "Si la Société de Jonage, autorisée à distribuer de "l'électricité pour l'éclairage, doit être imposée tout à la fois " comme exerçant les deux professions de loueur de force " motrice et d'exploitant une usine pour l'éclairage par l'élec" tricité, ou bien, si la distribution, faite par elle de l'électri" cité pour l'éclairage ne constitue, dans les conditions où elle " s'effectue, que des opérations accessoires de son industrie " principale ne pouvant donner lieu à des droits de patente " particuliers".

Le Conseil d'Etat admit ce système qui, d'ailleurs, était commandé par les principes du droit. S'il n'y a pas de tarif exactement applicable à l'énergie électrique, la classe la plus voisine est évidemment la location de force motrice; en matière fiscale, l'analogie porte sur la véritable nature de l'objet vendu et non pas sur le moyen de production de cet objet. Que la force provienne de l'électricité, qu'elle provienne d'un autre moteur, qu'elle soit lancée à distance ou consommée sur place, c'est toujours de la force. Du moment qu'il y a une catégorie de "loueur de force motrice ", l'énergie électrique doit y rentrer. Si l'usine produit de la lumière, elle rentre dans la classe de distribution d'éclairage électrique; si elle est mixte, il $y$ a lien de rechercher quelle est la principale exploitation pour savoir si l'éclairage constitue un simple accessoire et, dans le cas contraire, on doit appliquer les deux tarifs proportionnellement à chaque production.

Nous donnons ci-dessous le texte de l'arrêt (I); on remarquera sa date : il a été prononcé le I 2 juillet 1905 , presque en même temps que le vote de la loi de Finances promulguée

(i) Du r 2 juillet 1905

La 1 re sous-section du Cantentieux du Conseil d'Etat, siégeant $\in \mathrm{n}$ séance publique.

$\mathrm{Vu}$ les deux requêtes présentées par la Société Lyonnaise des Forces motrices du Rhône, tendant à ce qu'il plaise au Conseil, annuler deux arrêtés, en date du 30 décembre rgoz, par lesquels le Conseil de Préfecture du Rhône a rejeté ses demandes en réduction de la Contriburion des patentes à laquelle la dite Société a été imposée pour les années I001 et 1902 sur les rôles de la commune de Villeurbanne, par application d'un arrêté du Préfet du département du Rhône, du 23 août I 903 , en qualité d'exploitant une usine pour la production de l'électricité.

Ce faisant, attendu, en la forme..

$\mathrm{Au}$ fond que la profession exerce par la Société est calle prévue au tarif sous la dénomination de "loueur de force motricen, qu'il y avait lieu, dès lors, pour le Préfet, de prendre un arrêté d'assimilation; qu'in outre, la distribution de la force motrice ne présente aucune analogie avec la fourniture de l'éclairage; que si la Société a été au orisée à distribuer de l'énergie électrique pour l'éclairage: cette distribution, dans les conditions où elle est effectuée. ne constitue qu'un accessoire de l'industrie principale de Fa Société. Ordonner qu'elle sera imposée en qualité de loueur de force motrice ou, subsidiairement, à la fois en cette dernière qualité et en celle d'exploitant une usine d'éclairage par l'electriciré, proportionnellement à limportance relative des diverses opérations effectuées.

Vu la loi du 5 juillet $x 8$ So et les tableaux y annexés.

Vu la loi da 29 mars 1897 .

Sans ing toil

Sans qu'il soit besoin de statuer sur les moyens de forme relevés dans la requête;

Considérant que la distribution de l'ćnergie électrique pour l'éclai- fin juillet qui, conformément au texte ci-dessus donné, fixe la nouvelle taxation :

Il est donc inutile de répéter qu'il ne peut intéresser que les réclamations comprises dans le délai dont le point de départ est la date du premier arrêté d'assimilation (août Igoo) et le terme extrême, le 3 I décembre 1905.

Est-ce à dire que si cet arrêt était intervenu bien antérieurement à la loi, le pouvoir législatif en aurait été ému et aurait suivi, dans la loi nouvelle, la thèse du Conseil d'Etat, en assimilant d'un seuli coup pour l'avenir l'énergie électrique transportée au loin à la force motrice actuellement distribuée presque sur place dans dis proportions évidemment plus modes tes? La Chambre, en d'autres termes, aurait-elle consenti à faire tomber d'un seul coup, dans le tableau A, - le tableau des taxations relativement basses - cette industrie de l'énergie électrique que l'Administration, depuis Igoo, taxait au tarif le plus élevé?

Cette observation n'a pas encore été faite et l'on peut supposer, bien que lau naïveté ne perde jamais de ses droits, qu'elle ne viendra à l'esprit de personne; on comnât trop aujourd'hui, dans le monde industriel, labîme qui existe entre le milieu judiciäre et le milieu législatif. - Ce dernier n'a pas l'habitude de se laisser dicter des ordres par le premier, et l'Administration, malgré son échec au Conseil d'Etat, n'en aurait pas moins proposé au Parlement la tarification basée sur une assimilation entre la lumière électrique et l'énergie électrique. Peut-être niy eut-elle trouvé que des motifs pour insister davantage sur ce tarif à I franc le kilowatt qui, avant l'étude approfondie de la question, lui paraissait si précieux et si inattaquable. Tout le monde sait combien elle y tenait, excepté bien entendu, tous ceux qui n'ont pas eu de contact avec elle pendant l'ère des études et des difficultés.

Il ne s'agit pas, évidemment, d'un dégrèvement colossal : les extrêmes sont impossibles à obtenir. Il est intervenu seule-

rage et la fourniture de lélectricité comme force motrice constituent deux professions distinctes et ne présentent entre elles aucune analogie permettant de les assimiler au point de vue de l'imposition a la contribution des patentes :

Considérant que le tarif des patentes mentionnt, au nombre des professions imposables aux droits du tableau $A$, celle de loueur de force motrice; qu'il spécifie, il est vrai, que cette qualification est applicable ä celui qui, possesseur de bâtiments et de moteurs quiil nemploie pas pour son propte compte, les loue à divers industrits auxquels il s'engage à fournir une force motrice determiné, mais que la fourniture de la force motrice à autrui n'est prevtre par aucune autre disposition du tarif; que, dans ces circonstances, cette fourniture, alors même qu'elle n'aurait pas lieu dans les conditions spécialement définies au tarif, ne peut donner lieu, en l'état de la législation, qu'aux droits afférents actuellement a la profession de loveur de force motrice ;

Qu'il suit de là que la Scciété requérante, qui a été constituée dans le but de donner de la force motrice, est fondée à soutenir que c'est à tort qu'elle a été imposée, à raison de cette distribution, aux droits aftérents à la profession d'exploitant une usine d'éclairage par l'électricité, en conformité d'un arrêté préfectoral d'assimilation, et qu'elle n'est imposable, a raison de la fourniture de force notrice faite par elle aux particuliers, qu'en qualité de loueur de force motrice;

Considérant, il est yrai. que la Société a été autorisée à distribuer de l'électricité pour l'éclairage, mais que l'état de l'instruction ne permet pas d’apprécier dans quelle mesure elle a usé de cette autorisation et de décider si elle doit être imposée, tout à la fois comme exercant. les deux professions de loueur de torce motrice et d'exploitant une usine pour l'éclairage par Jélectricité, ou bien si la distribution faite par elle de lélecticicité pour l'éclairage ne constitue, dans les conditions où elle s'effectue, que des opérations accessoires de son industrie principale ne pouvant donner lieu à des droits de patente particuliers: Décide :

ro. - Arrêté annulé.

$2^{\circ}$ - La Société est renvoyée par devant l'Administration pour le réglement des droits de patentes auxquels elle doit être assujettic, en exécution de la présente décisionet, en cas de contestation, devant le Conseil de Préfecture.

30. - Rejet du surplus

$4^{\circ}$. - Timbre rembourse (4fr. 80 ). 
ment une disposition plus conforme que l'ancien état de choses à l'équité vulgaire. Cc tarif pourrait être analysé sous forme de dialogue entre l'Administration et le producteur d'électricité. Le contrôleur dit à l'usinier : "Combien de kilowatts " produisez-vous à vos machines, non comprises celles de se" cours? Sur ce total, combien en donnez-vous à la lumière? « Si c'est l'intégralité de la production, payez go cent.; si " ce n'est qu'une fraction, établissez-la; plus il y aura d'éner"gie, moins vous paierez cher (60 ou 30 centimes)". Cette réforme a été obtenue malgré un tarif proposé par l'Administration, distribué en projet à la Chambre, ne faisant que reproduire l'arrêté d'assimilation de huit ou dix Préfets de l'Est, du Sud-Est et du Midi.

Et comme le disaient les deux députés dont nous avons cité les noms, "si l'on veut tarifer l'énergie électrique d'une façon spéciale, au moins qu'on ne la frappe que pour ce qu'elle peut. donner ").

La loi de Finances a adopté ce système pour l'avenir.

Pour le passé, c'est-à-dire pour les quelques réclamations en cours, le Conseil d'Etat l'a aussi adopté, en déclarant fausses les assimilations faites par les Préfets.

Voilà où nous en sommes et la présente note n'a pas pour but d'en dire davantage.

Paul BOUGAULT, Avocat à la Cour d'Appel de Lyon.

\section{ÉTUDE SUR LES}

\section{BARRAGES EN MACGONNERIE}

\author{
et Murs de Réservoirs \\ (suite)
}

Barrage de Bouzey. - Nous dirons ici quelques mots du barrage de Bouzey, car c'est en recherchant les causes qui amenèrent sa rupture que M. Maurice Lévy a été amené à établir la méthode qui a fait l'objet de sa communication à YAcadémie des Sciences du 5 août 1895.

Le barrage de Bouzey avait été établi en travers de la vallée de l'A vière, petit affluent de la Moselle, pour créer un immense réservoir de 128 hectares de superficie et d'une eapacité de 7 millions de mètres cubes destinés à l'alimentation du canal de l'Est. Disposé en plan suivant sur une ligne droite, il avait une longueur en crête de 525 mètres et une hauteur maxima de 22 mètres au-dessus des fondations.

L'avant-projet du barrage, établi en 1876, fut approuvé par décision ministérielle du 12 février 1877 , après avis du Conseil général des Ponts et Chaussées. Cet avant-projet prévoyait une digue en maçonnerie de 20 mètres de hauteur, avec possibilité de la surélever ultérieurement de 2 mètres. Toutefois, par une décision du 13 septembre 1880 , le Ministre des Travaux publics approuva la proposition des ingénieurs du département de construire immédiatement la digue à sa hauteur définitive, afin d'évitèr par la suite les travaux complementaires nécessaires pour une surélévalion ultérieure. Cette décision fut prise malgré avis délavorable du Conseil général des Ponts et Chaussées, avis simplement motivé par ce fait que « les besoins de la navigation ne nécessitaient pas cet exhaussement ".

Commencóe en 1878, la digue fut complètement terminée à Ta fin de 1880 , et le remplissage commença en novembre 1881, Les eaux atteignaient la cote de $368^{\mathrm{m}} 80$, c'est-à-dire étaient à 2 in 70 au-dessous du niveau maximum fixé à $371^{\mathrm{m}} 50$, lorsque le 13 mars 1884 se produisit un accident urès grave. Entre les points numérotés 108 et 243 , c'est-à- dire sur 135 mèlres, la digue se déplaça brusquement vers laval, tout en restant verticale, et en prenant une forme concave vers l'amont avec une flèche maxima de 0 m34. En même temps, l'ensemble des fuites, qui dès le début du remplissage atteignait 60 litres à la seconde, passa brusquement de 75 à 232 litıes. Bien qu'un pareil fait dùt paraître tout à fait anormal, aucune mesure immédiate ne fut prise pour remédier à cet état de choses, bien mieux, on continua le remplissage et les eaux s'élevèrent jusqu'à la cote $369 \mathrm{~m}$.

C'ost seulement à la fin de 1885 que lon commençà s'inquiêter. On vida alors le réservoir pour inspecter la digue et l'on procéda à des sondages qui montrèrent que le grès bigarré très fissuré, sur lequel la digue était construite, avait cédé et s'était dislor ué sur une hauteur de 2 à 3 mètres. On constata de plus que le terrain sous la digue était en certains points constitué par des roches tendres et perméables.

Les travaux de restauration, terminés en septembre 1889 , consistèrent dans l'ètablissement d'un massif de butêe $B$. portant la lar"reur primitive de la base de $11 \mathrm{~m} 35$ à $47 \mathrm{~m} 45$, et s'appuyant lui-même sur un sommier s de $6 \mathrm{~m}$. de hauteur sur 4 m 40 de base. Pour relier le massif de butée avec le corps principal de la digue, on arracha la maçonnerie du parement aval primitif qu'on entailla suivant des dents de. scie sensiblement parallèles au parement nouveau. On bourra de béton les fouilles faites sous la digue et lon établit un solin $s$ au pied du parement amont, à la jonction de la digue et du mur de garde. Sur ce solin, on établit un premier remblai en terre argileuse corroyén, puis, par dessus, on fit ui remblai de graviers et de pierrailles ainsi que sur le pavement aval. L'ingénieur en chef du département avait proposé d'établir, en outre, contre le parement aval, un remblai de $10 \mathrm{~m}$. de largeur en crête et arasé a $4 \mathrm{~m}$. en
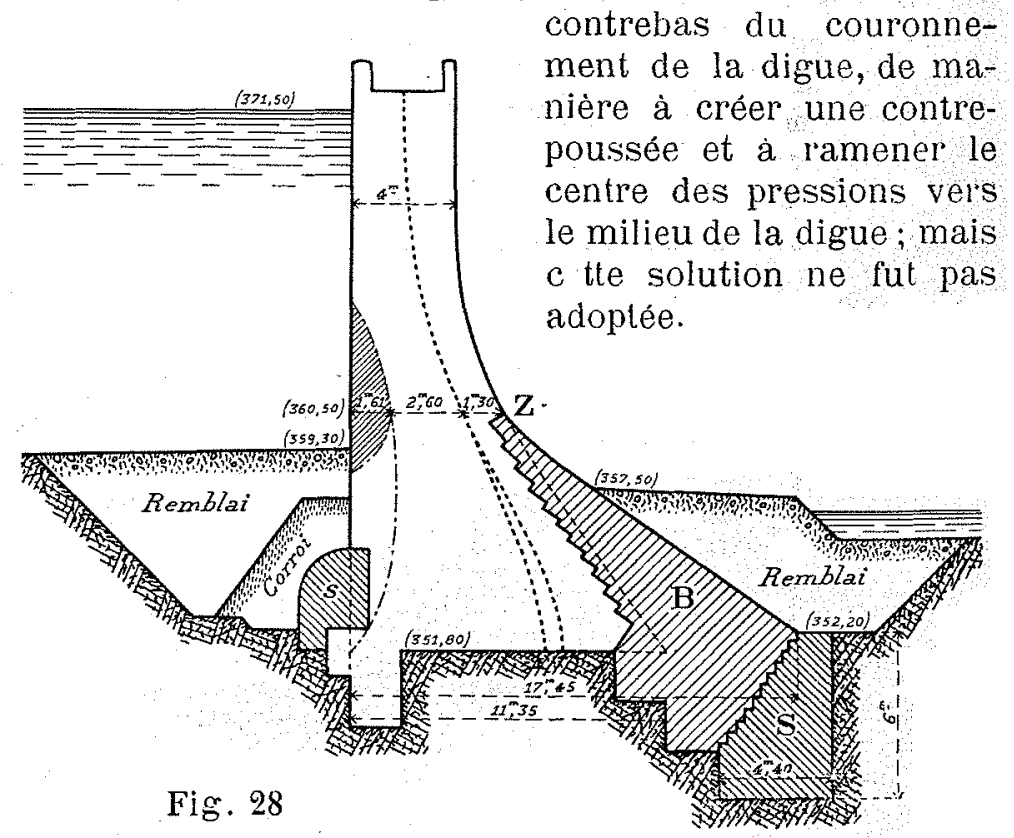

Le remplissage du réservoir commença deux mois après lachèvement des travaux et le niveau de leau ful porté jusqu'à la cote maxima de 371,50 .

Pour se rendre compte de la fièche maxima produite par la poussée de l'eau, on installa au début une lunette méridienne qui fut remplacée ensuite par un système de nivelettes. Gràce à ce dispositif on constata que la digue pouvait accuser une flèche de 18 millimètres par rapport à la posilion à vide. 